\title{
استراتيجية تمثيل الأدوار في تعليم اللغة العربية في القرن الحادي والعشرين
}

\author{
Akhsan Thayib \\ Universitas Ibrahimy Situbondo \\ ahsanthayyib@gmail.com
}

Tanggal Submitt: 15/11/2020

Tanggal diterima: $17 / 11 / 2020$

Tanggal Terbit: $27 / 6 / 2021$

$$
\begin{aligned}
& \text { ملخص: يتميز تطور القرن الحادي والعشرين باستخدام تكنولوجيا المعلومات والاتصالات في جميع جوانب الحياة، بما في ذلك } \\
& \text { عملية التعلم. يتطلب عالم العمل تغييرًا في الكفاءة. تعد القدرة على التفكير النقدي وحل المشكلات والتعاون كفاءات مهمة في } \\
& \text { دخول حياة القرن الحادي والعشرين. ويلزم أن تكون المدارس قادرة على إعداد الطلاب لدخول القرن الحادي والعشرين. تتكون } \\
& \text { موضوعات القرن الحادي والعشرين من اللغة الإنجليزية (اللغة الرسمية لكل بلد) واللغة الدولية والفن والرياضيات. والاقتصاد } \\
& \text { والمعرفة الطبيعية (العلوم) والجغرافيا والتاريخ والحكومة والمواطنة. تشمل موضوعات القرن الحادي والعشرين الوعي العالمي، ومحو }
\end{aligned}
$$

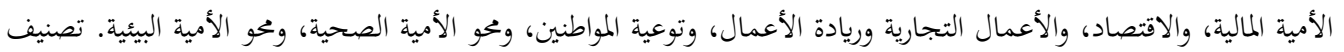

$$
\begin{aligned}
& \text { بلوم كمرجع في أهداف التعلم فيما يتعلق بأبعاد المعرفة والعمليات المعرفية. يشمل بُعد المعرفة الواقعي والمفاهيمي والإجرائي وما }
\end{aligned}
$$

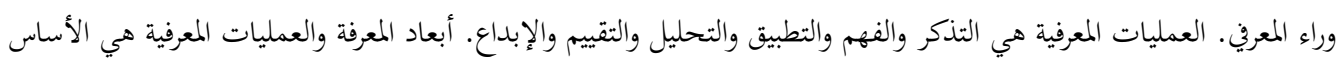

$$
\begin{aligned}
& \text { لتخطيط وتنفيذ وتقييم التعليم ، بحيث يتم بتميع استراتيجية التعليم للقرن الحادي والعشرين. } \\
& \text { الكلمات المفتاحية: مهارات القرن الحادي والعشرين، تصنيف الإزهار، استراتيجيات التعليم في القرن الحادي والعشرين. }
\end{aligned}
$$

\begin{abstract}
The development of the 21st century world is marked by the use of information and communication technology in all aspects of life, including the learning process. The world of work demands a change in competence. The ability to think critically, solve problems, and collaborate are important competencies in entering the life of the 21st century. Schools are required to be able to prepare students to enter the 21st century. Bloom's Taxonomy as a reference in learning objectives concerning the dimensions of knowledge and cognitive processes. Dimensions of knowledge include factual, conceptual, procedural, and metacognitive. Cognitive processes are remember, understand, apply, analyze, evaluate, create. The dimensions of knowledge and cognitive processes become the basis for planning, implementing and evaluating learning, so that 21st century learning strategies are arranged. In 21st Century Arabic Learning it has an impact on creativity to come up with learning strategies that are able to change students' willingness, interests, and talents in Arabic. The strategy that emerges is a role playing strategy (Role Mode), this strategy brain storming method is important to increase students' talents and interests in Arabic.
\end{abstract}

Keywords: Twenty-first century skills, classification, twenty-first century education strategies

مقدمة

$$
\text { الحياة. التكنولوجيا تربط العالم الذي يتجاوز الحواجز الجُغرافية والعشرين باستخدام تكنولوجيا المعلومات والاتصالات في جميع جوانب يصبح العالم بلا حدود. تسهل }
$$


تكنولوجيا النقل الجوي القيام برحلات طويلة. أصدر موقع beritasatu.com على الإنترنت وقتًا للسفر إلى

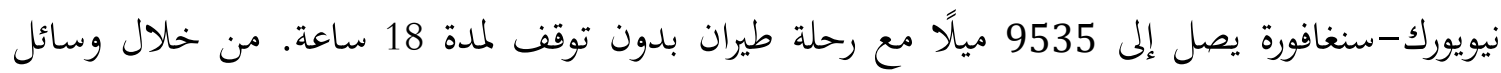

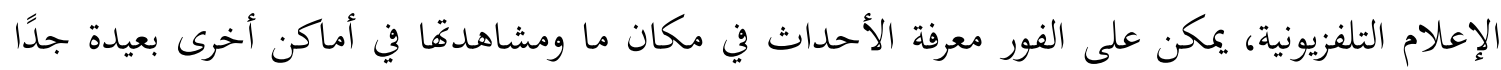
في نفس الوقت. إن تطوير تكنولوجيا المعلومات والاتصالات عبر الإنترنت يجعل من السهل إرسال الأموال في وقت قصير جدًا، حتى في الوقت الفعلي. تؤدي التطورات التكنولوجية إلى تغييرات في مؤهلات القوى العاملة وكفاءاتما.

لاحظ كم, كيم, و ييوا أن التغييرات في معايير الأداء الأكاديمي تحدث جنبًا إلى جنب مع تطور تكنولوجيا اتصالات المعلومات (ICT) والنمو الاقتصادي العالمي. تتطلب التغييرات في المعايير تعديلات في

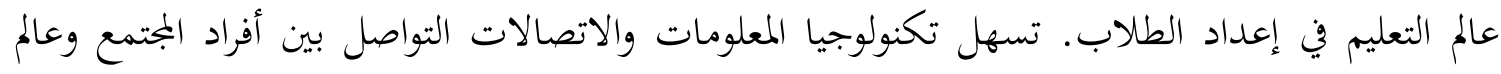
العمل الذي لا يقتصر على الزمان والمكان. يتطلب النمو الاقتصادي العالمي منافسة شرسة على نخو متزايد

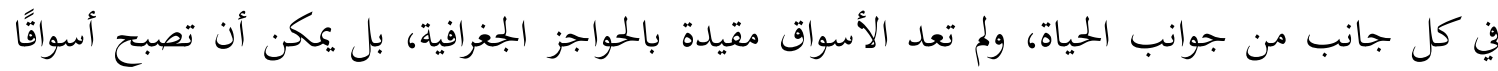
عالمية. يحتاج طلاب القرن الحادي والعشرين إلى أن يكونوا بجهزين بمهارات تكنولوجيا المعلومات والاتصالات وإيلاء اهتمام وثيق للتطورات الاقتصادية العالمية. يجب أن تستوعب عملية التعلم هذا. لاحظ روتيردام و ويليعهوم أن نجاح الطالب يعتمد على مهارات القرن الحادي والعشرين، لذلك يجب على الطلاب تعلم اكتساها. تحدد الشراكة لمهارات القرن الحادي والعشرين مهارات القرن الحادي

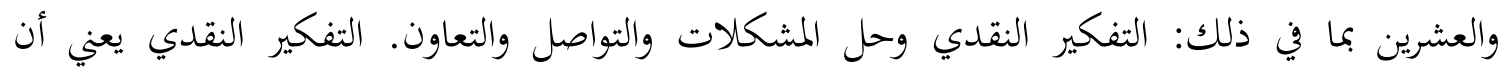
الطلاب قادرون على الاستجابة للعلم والمعرفة بشكل نقدي، وقادرين على استخدامها للإنسانية. المهارة في حل المشكلات تعني القدرة على حل المشكلات التي تواجهها في عملية أنشطة التعلم كوسيلة للتدرب على مواجهة مشاكل أكبر في الحياة. تشير مهارات الاتصال إلى القدرة على تحديد أدوات وتقنيات الاتصال

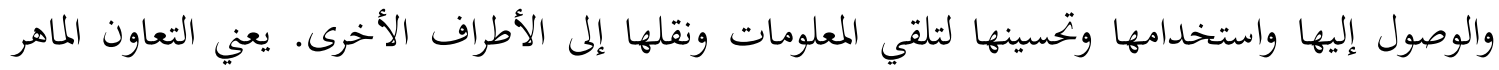

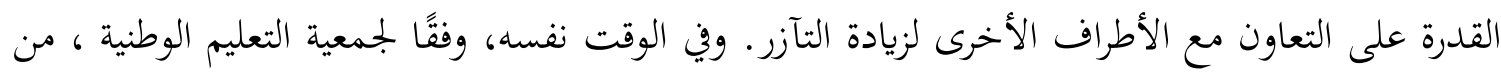

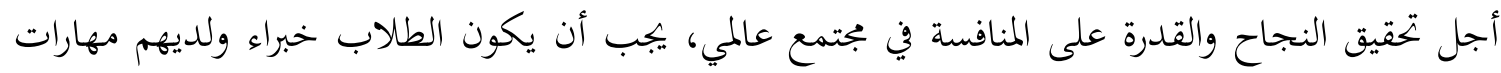
مثل التواصل والمبدعين والمفكرين النقديين والمتعاونين. استجابة لظاهرة تغيير احتياجات القوى العاملة والتقدم ، تحتاج المدارس إلى الاستعداد والاستعداد لمواجهة تحديات القرن الحادي والعشرين.من المهم نقل فهم مهارات القرن الحادي والعشرين للطلاب. يتم 
تحقيق مهارات القرن الحادي والعشرين من خلال فهم الخصائص وتقنيات الإنجاز واستراتيجيات التعلم التي يتم تنفيذها.

\section{مهارات القرن الحادي والعشرين}

تعتبر مسألة مهارات القرن الحادي والعشرين مصدر قلق للمراقبين التربويين والممارسين. يحدد مختبر التعليم الإقليمي الشمالي الأوسط (NCREL) وبجموعة (Metiri, 2003) إطارًا لمهارات القرن الحادي

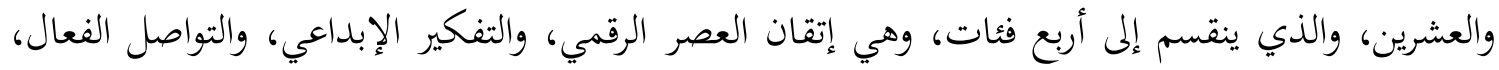
والإنتاجية العالية. يخلص ATCS (التقييم والتدريس لمهارات القرن الحادي والعشرين) إلى أربعة أشياء

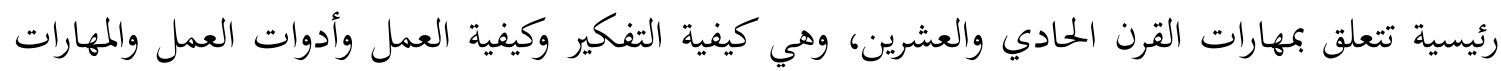

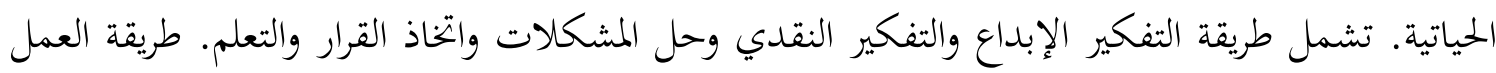
تشمل التواصل والتعاون. تشمل أدوات العمل تكنولوجيا المعلومات والاتصالات (ICT) ومحو الأمية المعلوماتية. تشمل المهارات الحياتية المواطنة والحياة والوظيفة والمسؤولية الشخصية والاجتماعية. تعرف خدمة الاختبارات التعليمية (2007) (ETS) مهارات القرن الحادي والعشرين على أفا تعلم

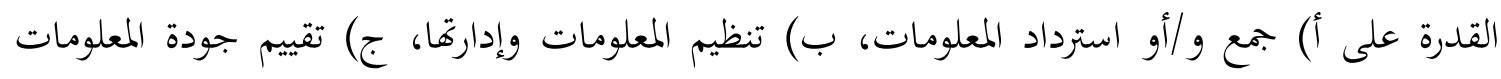

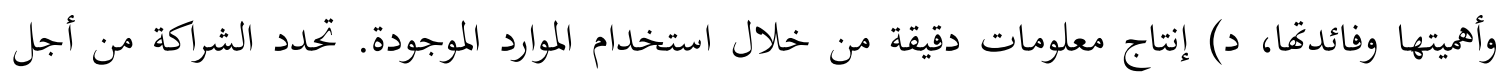

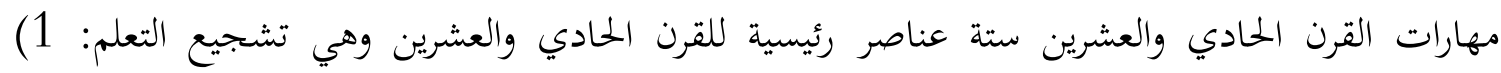

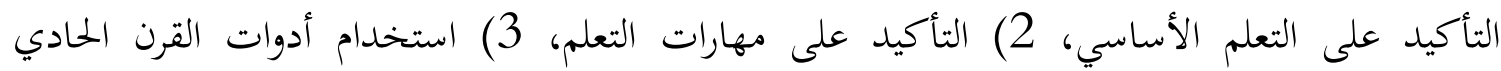

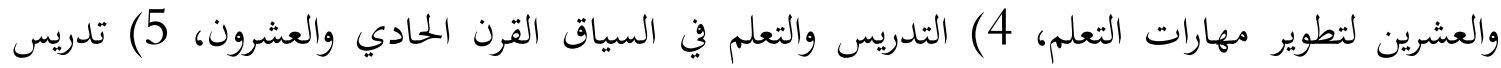
محتوى القرن الحادي والعشرين ودراسته، و 6) باستخدام تقييمات القرن الحادي والعشرين التي تقيس

$$
\text { مهارات القرن الحادي والعشرين. }
$$

يوفر كغ و كيم و يو إطارًا لمهارات القرن الحادي والعشرين في المجالات الثقافية المعرفية والعاطفية والاجتماعية. ينقسم البجال المعريف إلى بجالات فرعية: القدرة على إدارة المعلومات، أي القدرة على استخدام الأدوات والموارد ومهارات الاستفسار من خلال عملية الاكتشاف, القدرة على بناء المعرفة من خلال معالجة المعلومات وتقديم الأسباب والتفكير النقدي؛ القدرة على استخدام المعرفة من خلال عملية تحليلية 
وتقييم وتقييم وحل المشكلات, والقدرة على حل المشكلات باستخدام المهارات ما وراء المعرفية والتفكير الإبداعي.

يشمل المجال العاطفي المجالات الفرعية: الهوية الذاتية، أي القدرة على فهم مفاهيم الذات، والثقة

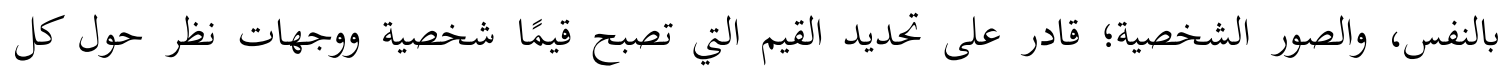
مشكلة. يظهر التوجيه الذاتي من خلال التحكم الذاتي والقدرة على التوجيه لتحقيق الأهداف في في إطار

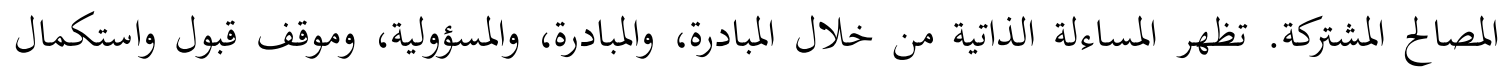

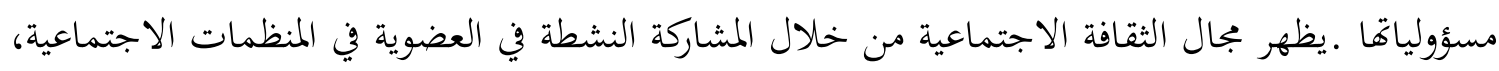
والقبول في البيئة الاجتماعية، والقدرة على الاختلاط الاجتماعي في البيئة.

\section{وموضوعات القرن الحمادي والعشرين}

إن فهم موضوعات القرن الحادي والعشرين وإتقانها يحدد نجاح الطالب في المستقبل. توفر الشراكة

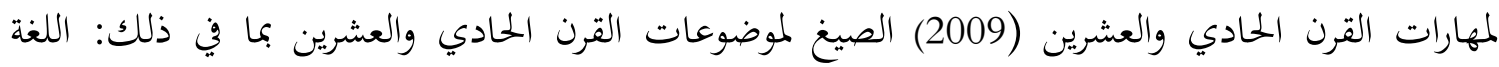
الإنجليزية (اللغة الرسمية لكل بلد)، واللغات الاجتماعية العالمية، والفن، والرياضيات، والاقتصاد، والمعرفة الطبيعية (العلوم)، والجغرافيا، والتاريخ، والحكومة، والمواطنة. يؤثر إتقان كل لغة وطنية ولغة العلاقات الدولية

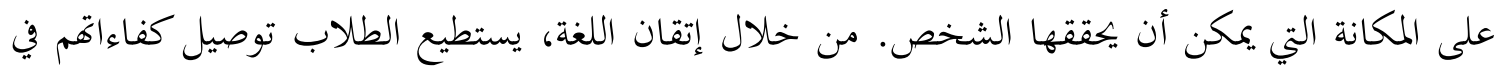

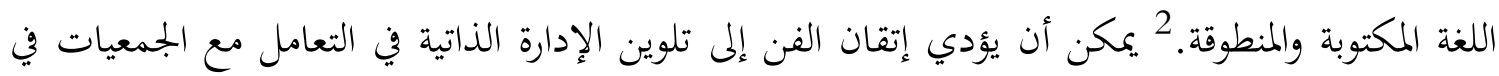

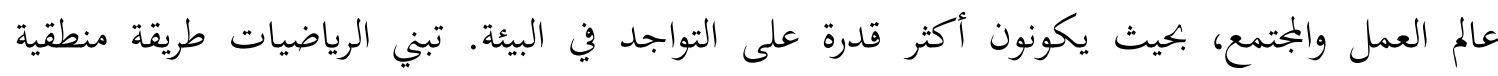
ومنهجية في التفكير، بحيث يمكن لإتقان الرياضيات أن يحسن منطق التفكير المطلوب للتفاعل. إن إتقان كفاءات هذه الموضوعات لم يكن له تأثير واسع على الطلاب إذا لم يكن مصحوبِّا بإتقان

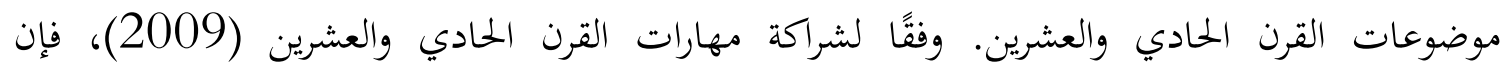
الموضوعات الناشئة في القرن الحادي والعشرين هي: الوعي العالمي, محو الأمية المالية والاقتصادية والتجارية

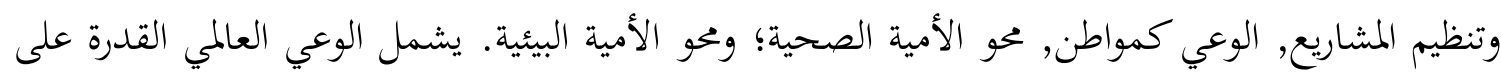
فهم القضايا العالمية والتعامل معها. القضايا العالمية في كل جانب من جوانب الحياة ، سواء كانت سياسية

${ }^{1}$ Kang, M., Kim, M., Kim, B., \& You, H. (n.d.), Developing an Instrumen to Measure 21st Century Skills for Elementary Student, 23

${ }^{2}$ Association, N. E. Preparing 21st Century Students for a Global Society : An Educator's Guide to the "Four Cs", 2001, 17 
أو اقتصادية أو اجتماعية أو ثقافية أو تكنولوجية أو معرفية. يعد التعلم من الأفراد الذين يمثلون مجموعة

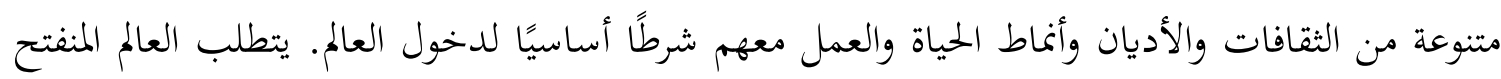
بشكل متزايد القدرة على قبول وفهم الجذور الثقافية والدينية ونمط الحياة للآخرين بروح الاحترام المتبادل

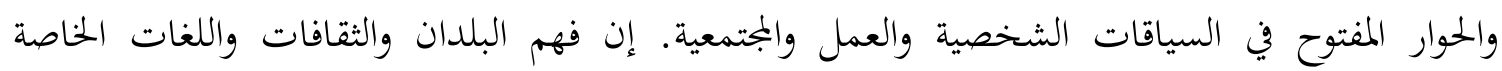

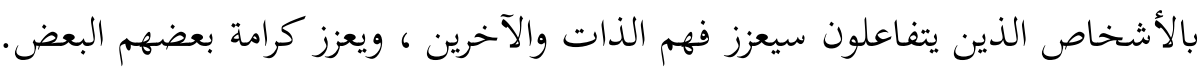

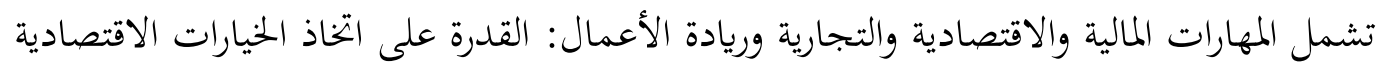

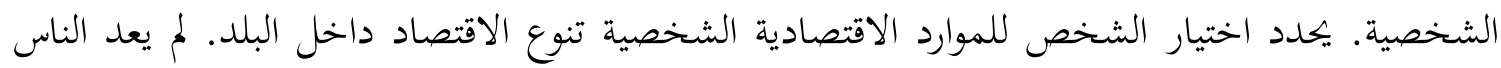
متأثرين بآراء الآخرين بشأن مواردهم الاقتصادية ، لكنهم يفسرون الموارد الاقتصادية على أخها وسيلة

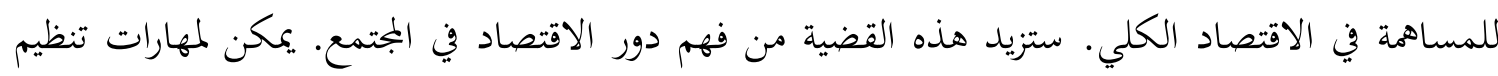
المشاريع لزيادة إنتاجية العمل والاختيارات المهنية أن تزيد من المساهمة في تطوير "المنظمة" التي تدخلهاديا.

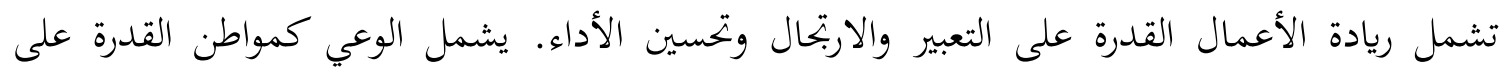

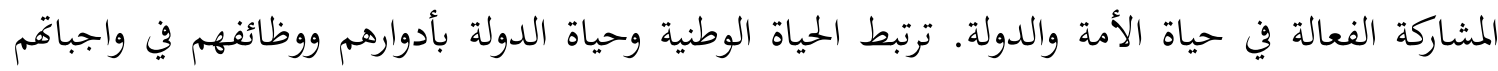

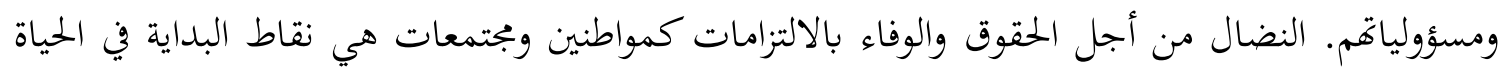

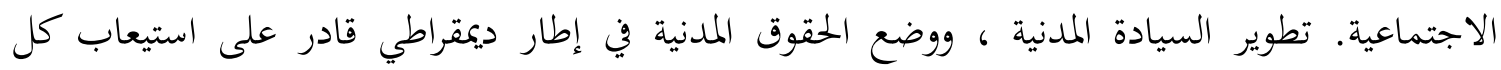
مصلحة فردية في تحقيق المصالح المشتركة.

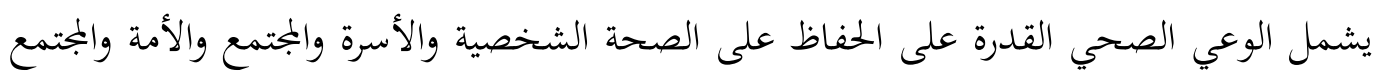
العالمي. تبدأ الرعاية الصحية بالقدرة على التماس المعلومات وتفسير المشكلات الصحية ، بما في ذلك الأسباب والتأثيرات وعملية الوقاية والعلاج. الصحة في هذا السياق هي الصحة الجسدية والعقلية بشكل

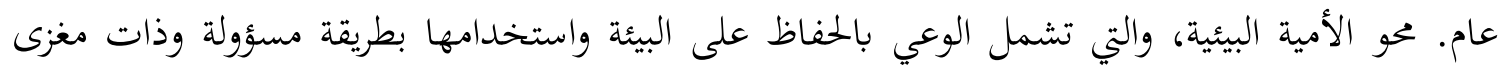

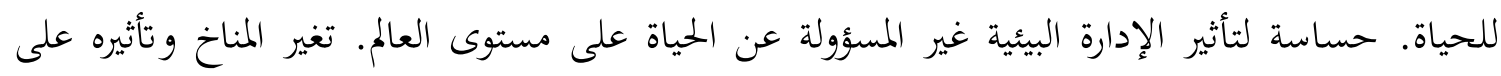
الحياة. التغيرات في السلوك الطبيعي التي تسبب تشوهات مناخية وتأثيرات على البيئة نتيجة الاستغلال

استراتيجيات التعليم في القرن الحادي والعشرين

يؤكد نموذج التعلم للقرن الحادي والعشرين على قدرة الطلاب على التفكير النقدي، والقدرة على

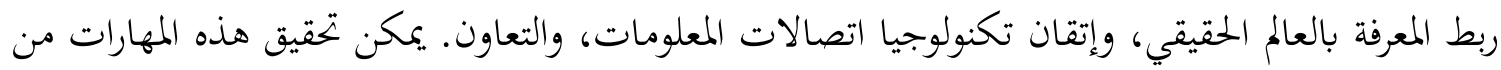


خلال تطبيق أساليب التعلم المناسبة من حيث إتقان المواد والمهارات. يتم بناء مهارات التفكير النقدي لدى الطلاب من خلال التعليم الذي يطبق تصنيف التعليم كما ذكر بنيامين بلوم في عام 1956 والذي تمت

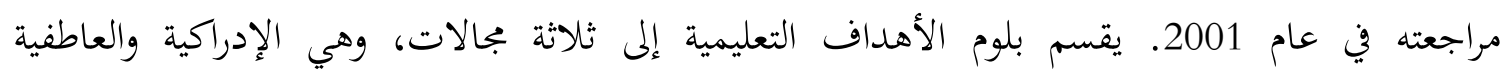
والنفسية. تم تنقيح الأهداف التعليمية في عام 2001. يصنف تصنيف التعلم إلى أبعاد المعرفة وأبعاد الادراديه

$$
\text { العمليات المعرفية3. }
$$

يتكون بُعد عملية المعرفة من أربعة أجزاء، هي الحقائق، والمفاهيم، والإجرائية، وما وراء المعرفية. يوضح أندرسون وكرتوال أن المعرفة الواقعية تؤكد على المعرفة الواقعية، وهي المعرفة في شكل أجلى أجزاء منفصلة من المعلومات أو العناصر الأساسية الموجودة في تخصص معين، والذي يتضمن معرفة المصطعلحة المعات والمعرفة. حول قسم التفاصيل. تقدم المعرفة الواقعية الحقائق التي تظهر في المعرفة. المعرفة المفاهيمية، وهي المعرفة التي

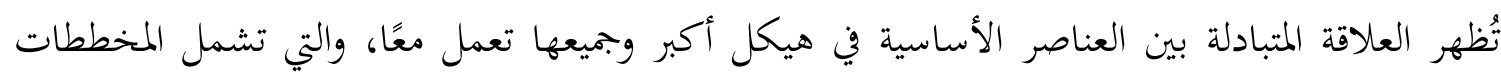

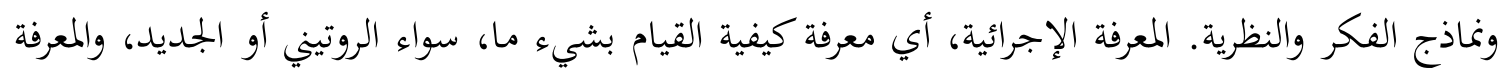

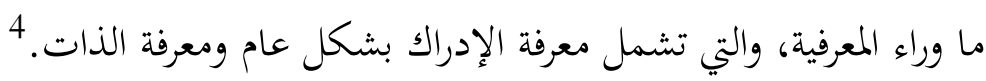
تنقسم أبعاد متلكات المعرفة إلى ثلاثة، وهي الإدراكية والعاطفية والنفسية الحركية ـينقسم البمال المعريف إلى ستة مستويات وهي: 1) تذكر: خذ واعترف وتذكر المعرفة ذات الصلة من ذاكرة المصطلح طويل؛ 2) الفهم (الفهم): بناء المعنى من الرسائل والرسومات المنطوقة والمكتوبة من خلاتلات وتلات التفسير والتمثيل والتصنيف والثلخيص والاستنتاج والمقارنة والشرح ؛ 3) تطبيق: تنفيذ أو استخدام الإجراءات من خلماءلال المنفذ، أو التطبيق ؛ 4) التحليل (التحليل): تقسيم المادة إلى الأجزاء المكونة لها، وتحديد ولهيد كيفية ارتباط الأجزاء ببعضها البعض والهيكل العام أو الغرض من خلال التمييز والتنظيم والتوصيل ؛ 5) التقييم (التقييم):

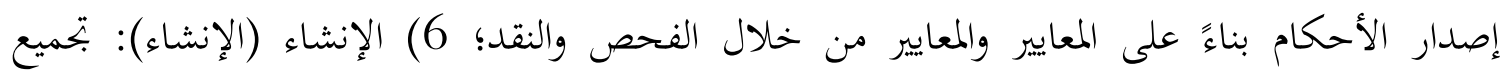

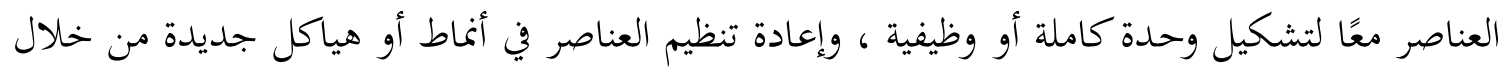

$$
\text { الإنتاج أو التخطيط أو الإنتاج. }
$$

لا يمكن أن تتم عملية التعلم القادرة على استيعاب مهارات التفكير النقدي لدى الطلاب من

خلال عملية التعلم أحادية الاتحاه. سيؤدي التعلم أحادي الاتحاه، أو المتمحور حول المعلم، إلى تقييد

3 Rotherham, A. J., \& Willingham, D. (2009). 21st Century Skills: the challenges ahead, Educational Leadership Volume 67 Number 1,16-21.

${ }^{4}$ Anderson, L. W., \& Krathwohl, D. R. (2001). A Taxonomy for learning, teaching, and assesing. a revision of Bloom's taxonomy of education objectives. New York: Addison Wesley Longman, 12 
انتقادات الطلاب عند الرد على مادة تعليمية. يتلقى الطلاب المواد من مصدر واحد، ويميلون إلى القبول

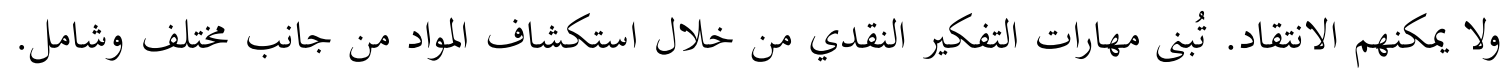
تتم القدرة على ربط المعرفة بالعالم الحقيقي من خلال دعوة الطلاب لرؤية الحياة في العالم الحقيقي. يعتبر

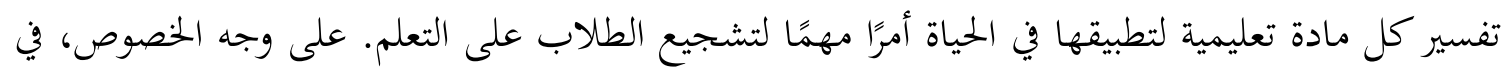

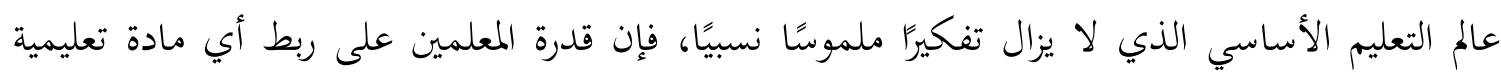

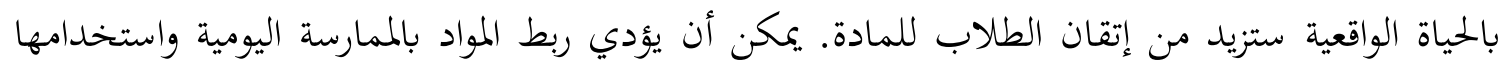
إلى تعزيز تنمية إمكانات الطلاب.

إن إتقان تقنية معلومات الاتصال أمر يجب أن يقوم به جميع المعلمين في جميع المواد. إن إتقان تكنولوجيا المعلومات والاتصالات الذي يحدث ليس على مستوى المعرفة، ولكن في ممارسة مستخدميها.

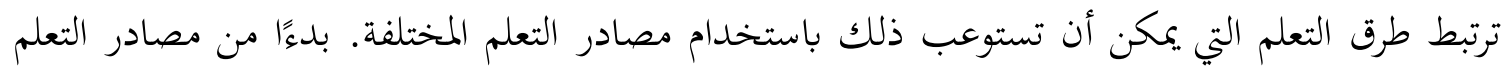

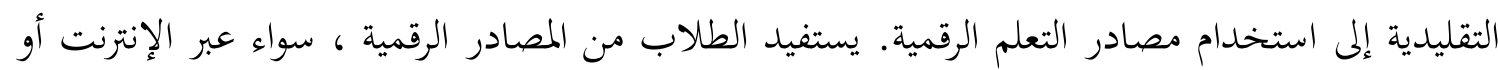
دون اتصال. ابتكار منتجات قائمة على تكنولوجيا المعلومات والاتصالات ، سواء سمعية أو سمعية بصرية. تظهر المهارات التعاونية قبول الآخرين والمشاركة مع الآخرين وتحقيق الأهداف المشتركة مع الآخرين. يسهل

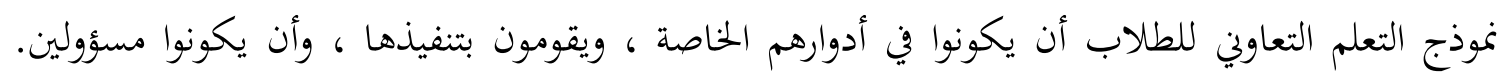

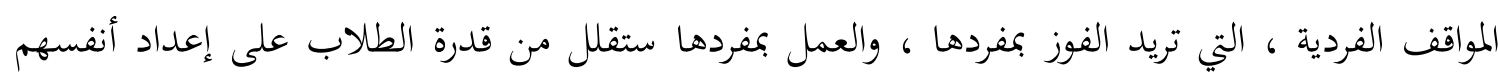
للمستقبل. يتم تعاون كل كفاءة موجودة في كل منها ، وذلك لزيادة الكفاءة وتحقيق النتائجّ ك.

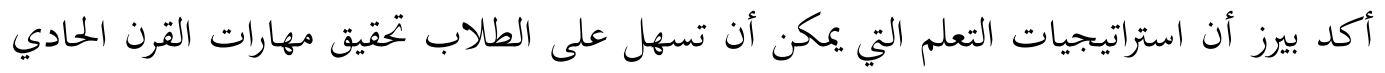

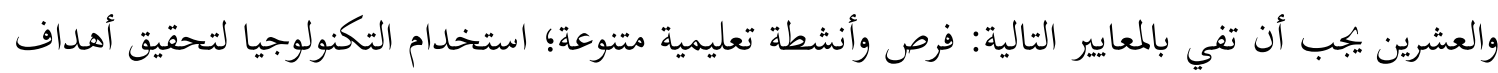

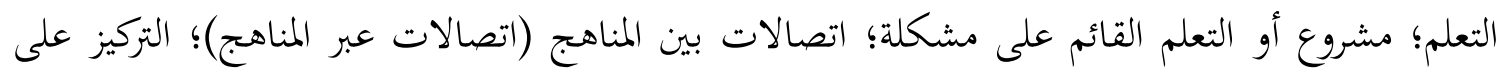

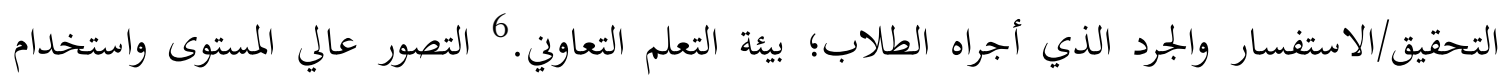

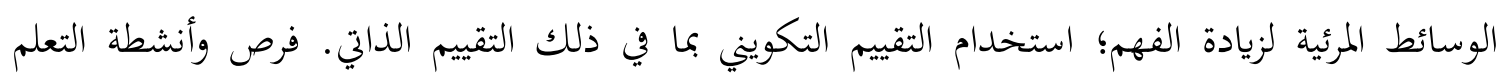

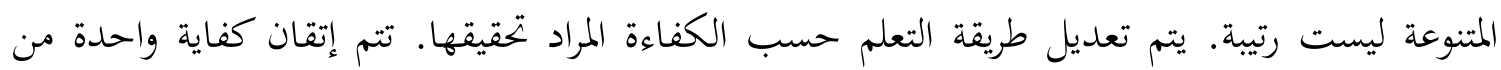
خلال بجموعة متنوعة من الأساليب التي يمكن أن تستوعب أنماط تعلم الطلاب السمعية والبصرية والجينية

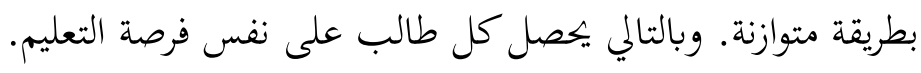

5 Krathwohl, D. R. (2002), A Revision of Bloom's Taxonomy: An Overview, THEORY INTO PRACTICE , 212-232.

${ }^{6}$ Beers, S. Z. (2012). 21st Century Skills: Preparing Students for THEIR Future, 15 
إن استخدام التكنولوجيا، وخاصة تكنولوجيا الاتصالات المعلوماتية، يسهل الطلاب على متابعة التطورات التكنولوجية، والخصول على أنواع مختلفة من مصادر التعلم والوسائط. تسمح مصادر التعلم

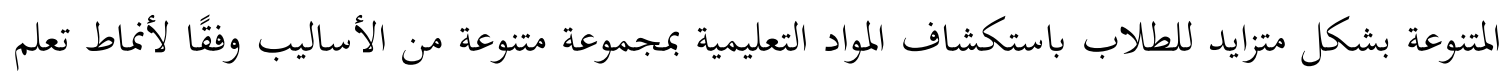

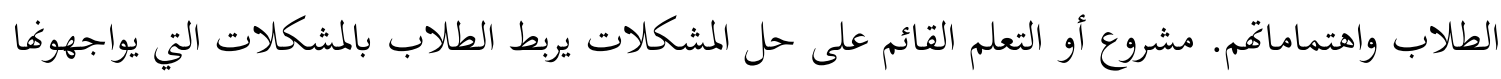

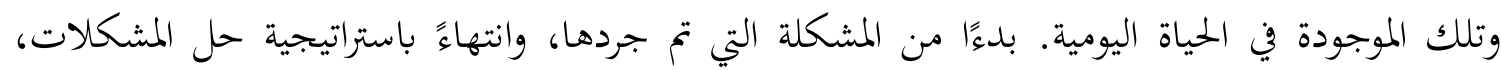
يتعلم الطلاب باستمرار المواد والكفاءات التعليمية المنظمة. في التعلم المعتمد على المشاريع، يتم التعبير عن فئن حل المشكلات في منتجات حقيقية يتم إنتاجها كعمل من إبداع الطلاب. في مشاريع التعلم/التعلم القائمة

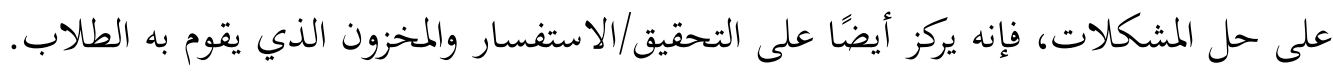

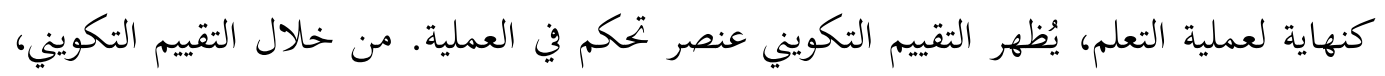

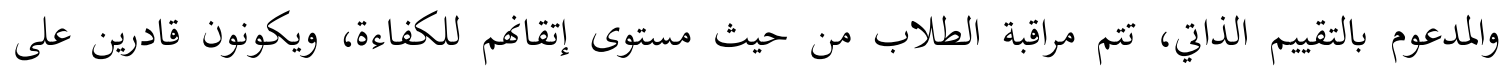

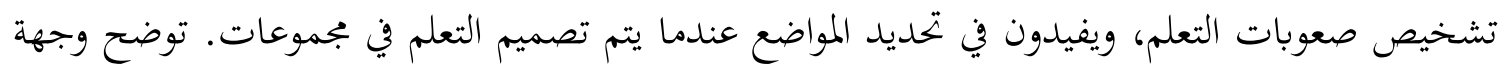

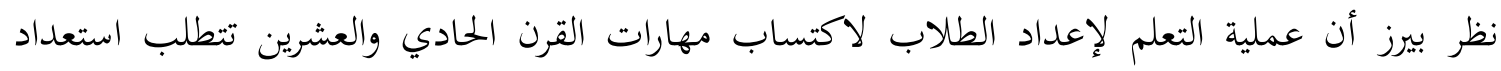
المعلمين لتخطيط وتنفيذ وتقييم التعلم. يلعب المعلم دورًا مركزيًا كميسر للتعلم. يتم تسهيل الطلاب في عملية إتقان المواد التعليمية مع مختلف مصادر التعلم المعدة. المعلم مسؤول عن الإشراف على العملية التي بتري في إطار إتقان الكفاءات، على الرغم من أن التعلم يتمحور حول الطالب.

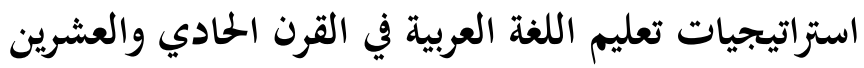

فإن تعليم اللغة العربية للناطقني بغريها يقصد به: سلوك المعلمين أثناء عملية تعليم اللغة العربية لإكساب الطالب الناطقين بغيرها الخبرات والمعلومات والمهارات والاتجاهات التي ينبغي تعلمها في مادة اللغة

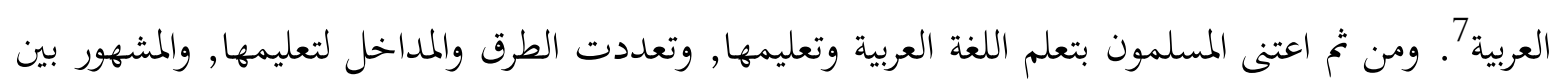

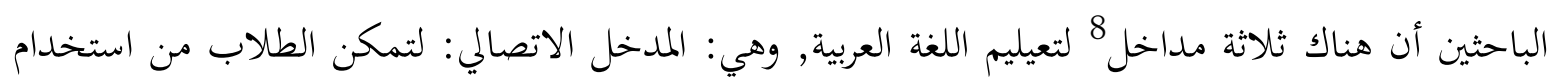

7 انظر تقويم تدريس مادة اللغة العربية، مشرق محمد العيساوي، العراق، جامعة بابل، كلية التربية الأساسية، بحث

$$
\text { منشور بتاريخ: 6/6 / 2011م. }
$$

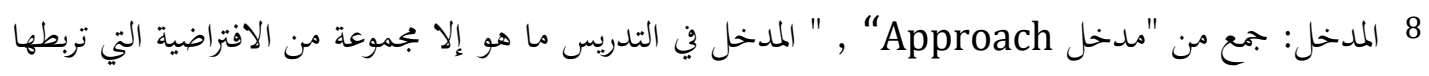
بعضها بعضا علاقة متبا دلة, هذه الإفتراضات تتصل اتصالا وثيقا بطبيعة اللغة وطبيعة عمليتي تدريسها وتعلمها....ولتوضيح ذلك نضرب مثلا, " المدخل السمعي الشفوي" وافتراضات اللغوية هي: اللغة إنسانية سمعية شقهية وهي رموز تحمل المعاني, وتختلف اللغات بعضها عن بعض, ويمكن كشف تركيب اللغة وبنائها ووصفها وصفا منظما ومفيدا. 
اللغة الاتصالية عن طريق المحادثة والكتابة, والمدخا التكاملي: الذي يعتبر أن المواد اللغوية كلها تكمل بعضها بعضا وذلك عن طريقة إتقان قواعد النحو والصرف والبلاغة والمحادثة والكتابة بمقتضى الحال, وأما المدخل

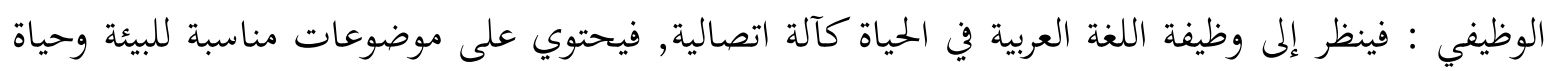
الطلاب الذين يتعلموها.

اختلف المتخصصون في مجال التدريس حول مفهوم الإستراتيجيات الحلديثة في التعليم, ويمكن أن

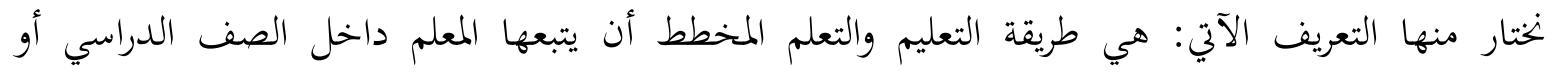
خارجهه, لتدريس محتوى موضوع دراسي معين, بغية تحقيق أهداف محددة سلفا, وينضوي هذا الأسلوب على النه

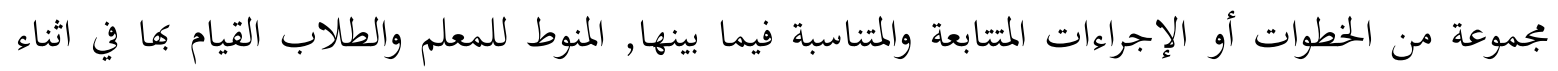

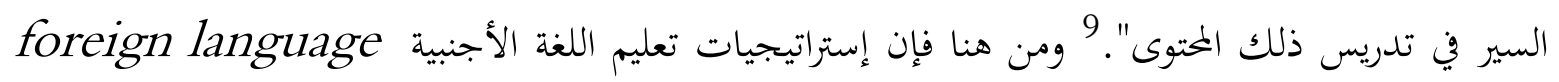
learning strategi وإتقاها, ومن شاها أن تسهل تعلم هذه اللغة, وتخفف الصعوبات التي فد تعرضه في تعلمها, وتحعله أكثر بعرة اعتمادا على نفسه. 10

\section{استراتيجية تمثيل الأدوار وتطبيقتها في اللغة العربية}

تعد " استراتيجيات تمثيل الأدوار" من أفضل إستراتيجيات تعليم اللغة العربية للناطقين بغيرها,

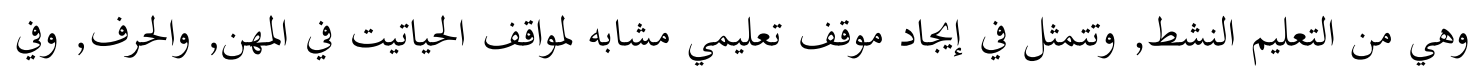

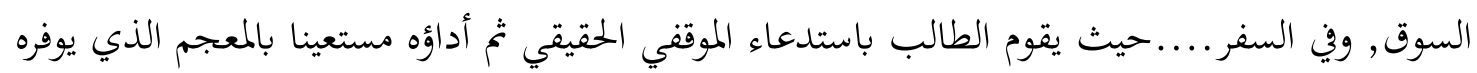

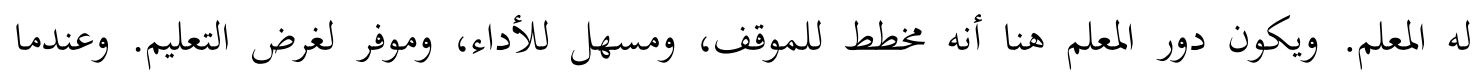

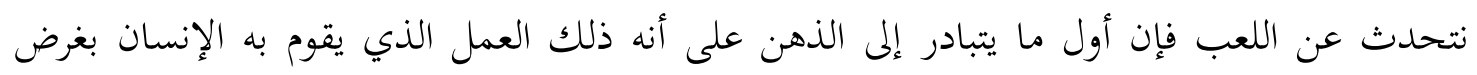
التسلية وتمضية الوقت، واللعب بهذا المعني يرتبط بالنواحي السلبية.

9 حسن حسين زيتون, إستراتيجيات التدريس: رؤية معاصرة لطرق التعليم والتعلم, القاهرة, ط, عالم الكتب, 5003

10 محمد علي الخوالدة, استراتيجيات تعليم اللغة العربية لدى الطلبة الماليزيين في جامعة اليرموك وعلاقتها بمتغيري

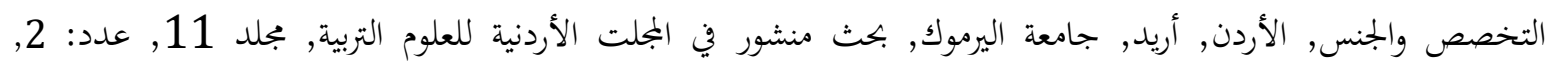
2015م, ص 171. 
وبخاصة عندما نتحدث عن التعليم والتعلم والعملية التربوية، إن هذا النوع من اللعب هو اللعب غير الواضح وغير موجة ولكن قد يكون اللعب موجها و هدفا وذا قيمة إذا ما استغل بطريقة صحيحة،

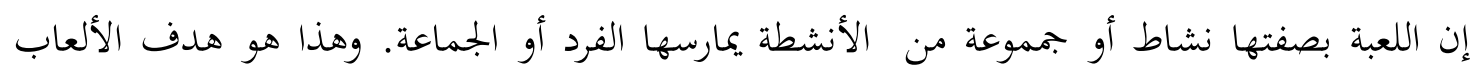
التعليمية فهي تستخدم بغرض تنمية سلوك الأطفال وشخصياتم و إنماء العقل عند الأطفال وتسهيل

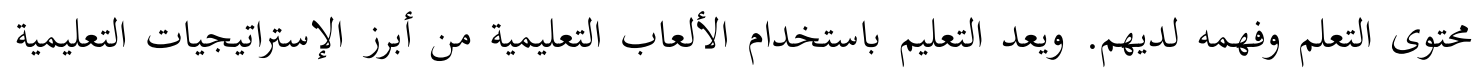
التي تراعي سيكولوجية المتعلمين. 11 عناصر إستراتيجية تمثيل الأدوار: 1. الأدوار: ففي اللعب أو اللعبة الواحدة يتم تحديد أدوار معينة للأفراد ذوي العالقة العة. 2. القواعد والقوانين: فاللعبة تسير وفق قواعد وقوانين حمدة بصورة مسبقة ويجري الاتفاق عليها من قبل الأفراد أو اللاعبين.

3. الأهداف: كل لعبة لها هدف أو بجموعة أهداف يسعى اللاعبون ويتنافسون لتحقيقها، فهدف لعبة كرة القدم مثلا هو تحقيق أكبر عدد مككن من الأهداف.

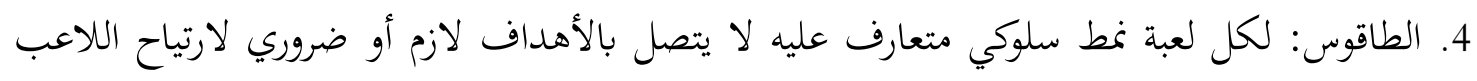

$$
\text { للعب, والاستمرار فيه. }
$$

5. اللغة: لكل لعبة مصطلحاتا أو قاموسها الخاص لا يتصل بالأهداف والقوانين ولكنها لازمة وضرورية

$$
\text { ويجب تعلمها واستعمالها. }
$$

6. القيمة أو المعيار : لكل لعبة معايير نجاح معينة أو قيمة معينة.

$$
\text { محاكات استراتيجية تمثيل الأدوار: }
$$

وهذا نوع آخر من لعب الأدوار أو تمثيلها وفيها يتصرف الأفراد أو الممثلين وكأفم في مجريات

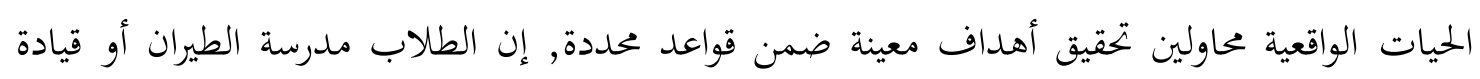

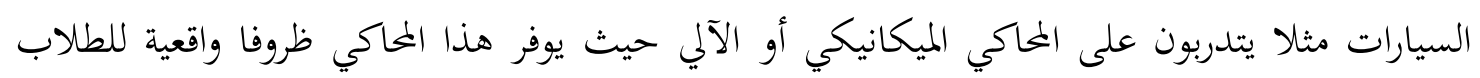
للتعلم على قيادة الطائرة أو السيارة.

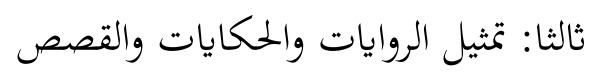

11 انظر طرق التدريس في القرن الواحد والعشرين، د. عبد اللطيف بن حسني فرج، الأردن، عمان، ط2 :دار

$$
\text { المسيرة، } 1426 \text { هـ, 2005م, ص. 56-36. }
$$


تمثيل الروايات والحكايات والقصص أمر مألوف لدي طلاب المدارس, ولا تقتصر عملية

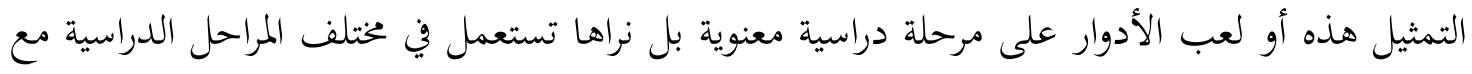

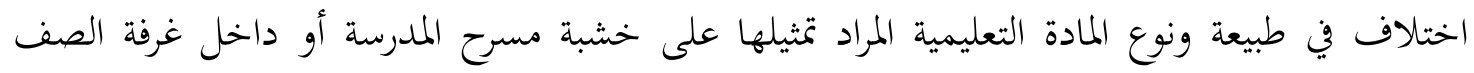

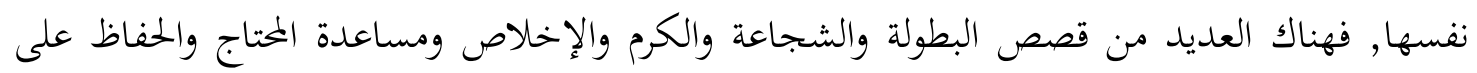
الجار التي يمكن للطلاب أن يتعلموها عن طريق تمثيلها. رابعا: أهمية طريقة تمثيل الأدوار

تتصل هذه الطريقة وما تتضمن من ألعاب ومحاكاة وتمثيل اتصالا مباشرا بحياة الطلاب وتعمل

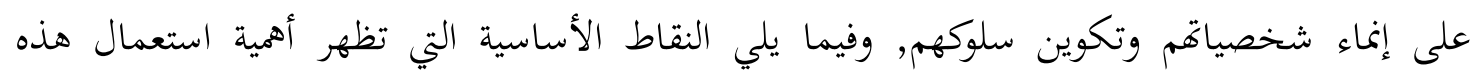

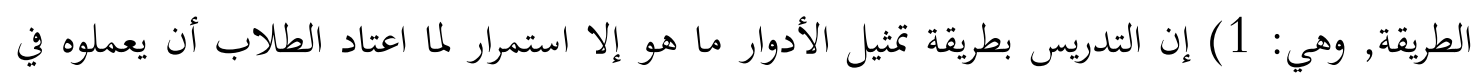

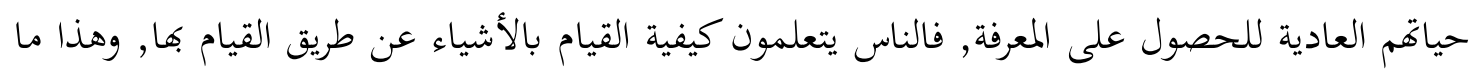

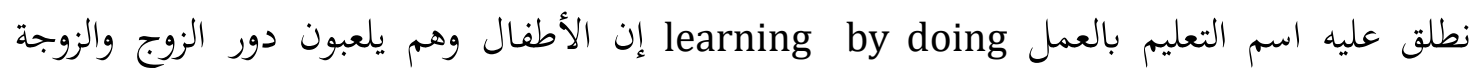

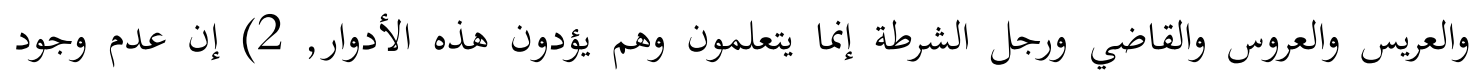

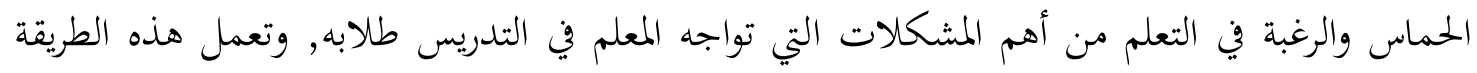

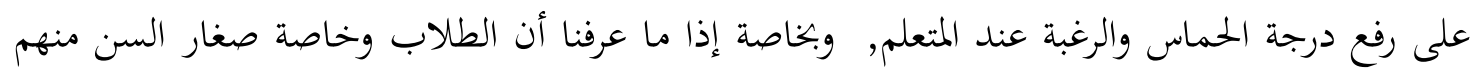

$$
\text { 2) اسمبون اللعب, وهم يتعلمون عن طريقه. }
$$

تعتبر "إستراتيجية العصف الذهني brain - storming methode " أحدث إستراتيجية تعليمية

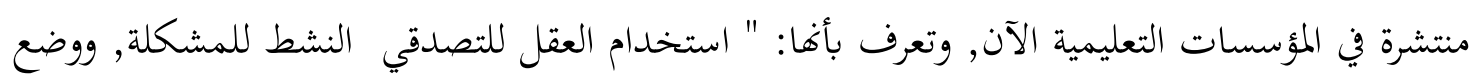

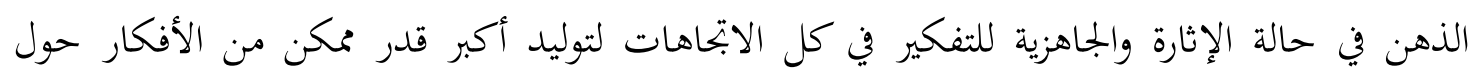

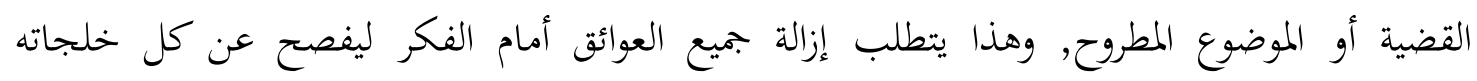

وخيالانه".

الحلاصة

12 خليل إبراهيم الحويجي, مهارة التعلم والتفكير, الأردن, عمان, ط: دار زمزم للنشر والتوزيع, 2012م, ص. 
استراتيجية تعليم اللغة العربية للقرن الحادي والعشرين هي استراتيجية تمدف إلى إثارة حماس الطلاب

ورغبتهم واهتمامهم بتعليم اللغة العربية (دراسة علم نفس التعلم)، بالإضافة إلى إثارة عقول الطلاب على

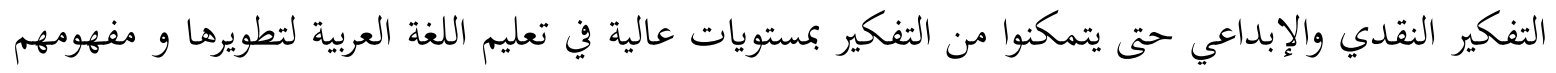
في التفكير.

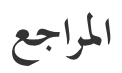

Anderson, L. W., \& Krathwohl, D. R. (2001). A Taxonomy for learning, teaching, and assesing. a revision of Bloom's taxonomy of education objectives. New York: Addison Wesley Longman.

Association, N. E. Preparing 21st Century Students for a Global Society : An Educator's Guide to the "Four Cs".

Beers, S. Z. (2012). 21st Century Skills: Preparing Students for THEIR Future.

Center, P. P. (2010). 21st Century Skills for Students and Teachers. Honolulu:: Kamehameha Schools, Research \& Evaluation Division.

Kang, M., Kim, M., Kim, B., \& You, H. (n.d.). Developing an Instrumen to Measure 21st Century Skills for Elementary Student.

Krathwohl, D. R. (2002). A Revision of Bloom's Taxonomy: An Overview. THEOR Y INTO PRACTICE , 212-232.

NCREL \& Metiri Group. (2003). enGauge 21st century skills: literacy in the digital age. http://www.ncrel.org/engauge/skills/skills.htm

Rotherham, A. J., \& Willingham, D. (2009). 21st Century Skills: the challenges ahead. Educational Leadership Volume 67 Number 1, 16-21.

Skills, P. f. Learning for the 21st century skills. Tucson,: Partnership for 21st Century Skills.

$$
\begin{aligned}
& \text { حسن حسين زيتون, إستراتيجيات التلدريس: رؤية معاصرة لطرق التعليم والتعلم, القاهرة, ط, عالم الكتب, } 2003 \text { م. }
\end{aligned}
$$

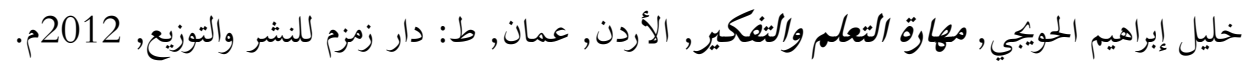


عبد اللطيف بن حسني فرج, طرق الثلديس في القرن الواحد والعشرين، الأردن، عمان، ط2 :دار المسيرة، 1426 هـ, 2005

محمد العيساوي, تقويم تلدريس مادة اللغة العببية، العراق، جامعة بابل، كلية التبية الأساسية، بحث منشور بتاريخ: 6/6 .2011/

محمد علي الخوالدة, استراتيجيات تعليم اللغة العربية لدى الطلبة الماليزبين في جامعة اليرموك وعلاقتها بمتغيري التخصص التصل

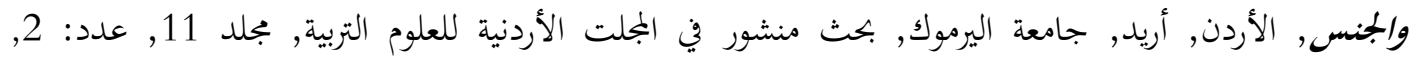
2015 